\title{
Optimum Lifetime Planning of Bridge Inspection and Repair Programs
}

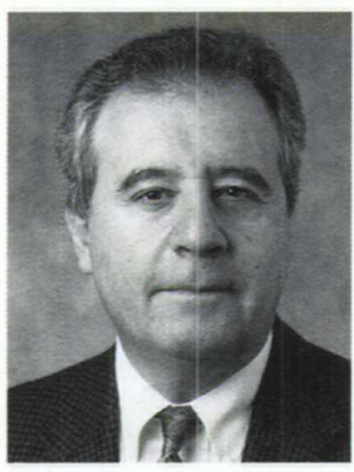

\section{Dan M. Frangopol}

Prof.

Univ. of Colorado

Boulder, CO, USA

Dan M. Frangopol received civil engineering degrees from the Institute of Civil Engineering, Bucharest, Romania, and the University of Liège, Belgium. After working in Belgium, he joined the University of Colorado in 1983. His research activities include reliability analysis, bridge management systems, and structural optimization.

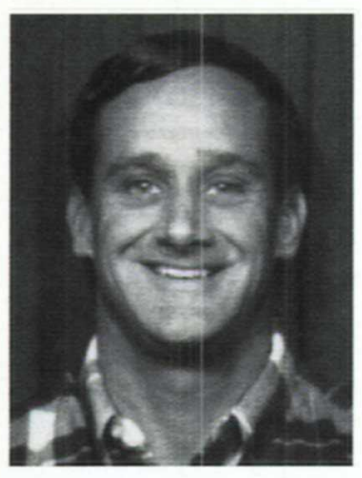

\section{Allen C. Estes}

Assoc. Prof.

United States Military Academy

West Point, NY, USA

Allen C. Estes obtained his M.S. from Stanford University and his Ph.D. from the University of Colorado. In 1999, he was appointed Associate Professor at the US Military Academy in West Point. His research activities include system reliability, and optimization of inspection and repair programs.

\section{Summary}

This study proposes a general methodology for determining the optimal lifetime planning of bridge inspection and repair programs based on minimizing the expected cost while maintaining an acceptable level of reliability. For individual bridges, this methodology determines the optimum inspection technique, and the numbers and timing of inspections and repairs. The methodology is demonstrated initially on a simple structure. Then, the method is applied to the optimal planning of inspection and repair programs for an existing bridge. The proposed approach to the problem of lifetime bridge maintenance is shown to be a viable method for optimizing inspection and repair investments during the expected remaining life of existing bridges. The results have important implications in the development of future reliability-based maintenance guidelines and criteria for the inspection and repair of bridges.

\section{Introduction}

The goal of optimal lifetime planning of bridge maintenance is to determine and implement the best possible strategy for allocating limited resources to the inspection, maintenance, rehabilitation and replacement of bridges. The optimum strategy has to achieve a balance between lifetime reliability and expected life-cycle cost. Although this is generally recognized for various structural applications [1-4], including bridges [5], and there has been progress in bridge reliability and bridge life-cycle cost, the integration of bridge lifetime reliability analysis with bridge life-cycle cost analysis has been very limited. In the USA, it was recognized that "while the quality and performance of infrastructure are vital to the nation's economic and social well-being, by most accounts this investment has not been prudently managed for sustainability. New knowledge is needed to provide the intellectual support for infrastructure decisions necessary to sustain economic growth" [6].

The main objective of this study is to contribute to the process of optimal bridge management by proposing a methodology for determining the optimum inspection and repair programs for existing bridges based on minimizing the expected cost while maintaining an acceptable level of reliability. In this manner, the limited available resources are managed in an optimal manner, both in economic and reliabil- ity terms. This proposed methodology determines the optimum inspection technique, the numbers of lifetime inspections and repairs, and the timing of these inspections and repairs for an individual structure. This methodology does not address the planning of ordinary inspections, such as the biennial visual bridge inspections required in the USA. The paper focuses on integration of non-destructive evaluation techniques, such as acoustic emission, radar, infrared thermography and halfcell potential, in bridge management.

\section{General Methodology}

The general methodology for optimizing the lifetime inspection/repair strategy for a structure is as follows [7,8]:

- define the structure and the criterion that constitutes failure of the structure

- specify how the structure deteriorates over time and develop a deterioration model

- specify the inspection methods available to detect this deterioration and quantify the detection capability and cost of these methods

- define the available repair options and calculate their costs

- quantify the probability of making a repair if a defect is detected

- formulate the optimization problem based on the optimization criterion, 
failure constraints, expected life of the structure, minimum and/or maximum time intervals between inspections, etc.

- use an event tree to account for all of the repair/no repair decision possibilities that must be made after every inspection

- optimize the timing of a given number of lifetime inspections for a specific inspection technique

- repeat the problem for different numbers of lifetime inspections and inspection techniques to find the optimum strategy

- update the optimum strategy after every inspection using the new information provided from the inspection results.

In this paper, the methodology is applied first to the lifetime inspection/repair optimization of a simple deteriorating structure, then to existing bridges in a whole-life perspective.

\section{Simple Deteriorating Structure}

The simple deteriorating structure under consideration has been defined in [7]. Its resistance $(R)$ and load $(P)$ are considered time-independent random variables, while its deterministic crosssectional area $(A)$ is assumed to vary with time. The criterion that constitutes failure of the structure is $R \leq P / A(t)$, where $A(t)$ is the time-variant cross-sectional area. The deterioration of the cross-sectional area over time, assuming that no repairs are made [7], is

$$
A(t)=A_{\text {initial }}-0.102 t^{0.57}
$$

Fig. 1 shows the deterioration of the cross-sectional area, for the case $A_{\text {initial }}$ $=1.0$. Due to the rapid deterioration process only half of $A_{\text {initial }}$ remains after about 17 years.

Given the simple deteriorating structure defined by the main descriptors

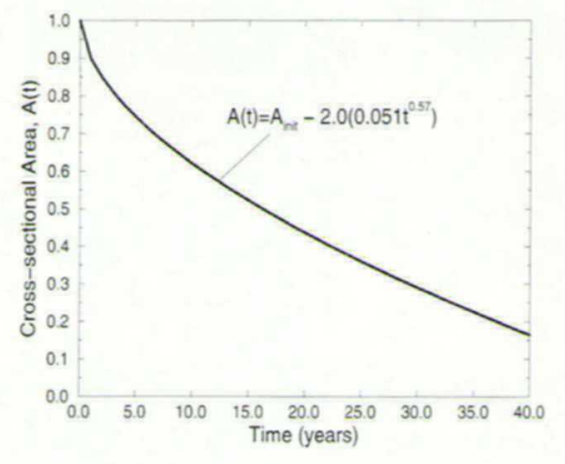

Fig. 1: Deterioration over time

of resistance $[\bar{R}=14.0$ and $\sigma(R)=1.4]$ and load $[\bar{P}=8.0$ and $\sigma(P)=0.8]$, where $\bar{x}$ and $\sigma(x)$ are the mean value and standard deviation of $x$, respectively, the goal is to develop a strategy that will minimize the expected total cost $\left[E\left(C_{\text {tot }}\right)\right]$ of the lifetime inspection/repair program and prevent the structure from deteriorating to an unacceptable level of reliability at any point during its service life. The assumed service life of this structure is 10 years, and the expected value of the reliability index $[E(\beta)]$ will not be permitted to fall below $\beta_{\min }=2.0$. There will be two, three or four inspections allocated over the life of the structure. The design variables are the inspection technique and the inspection times.

Four inspection techniques (A, B, C and D) with normally distributed damage-detection capabilities are considered (Table 1). The ability of these methods to detect damage is based on the intensity of the structural damage $\left(\eta_{\text {str }}\right)$, which is related to the section loss as follows:

$$
\eta_{\text {str }}(t)=\left[A_{\text {initial }}-A(t)\right] / A_{\text {initial }}
$$

The main descriptors, including costs, associated with the four inspection techniques are shown in Table 1, where $\eta_{0.5}$ is the damage intensity at which there is a $50 \%$ chance of detection, $\sigma_{\text {insp }}$ is the standard deviation of the detection ability of the inspection, $\eta_{\min }$ is the damage intensity below which detection is impossible, and $\eta_{\max }$ is the damage intensity above which detection is absolutely certain. The values for $\eta_{\min }$ and $\eta_{\max }$ correspond to three standard deviations below and above $\eta_{0.5}$, respectively [9].

The probability of a defect being detected $\left(P_{\text {det }}\right)$ at time $t$ is dependent on the damage intensity of the structure at that time $\left[\eta_{\text {str }}(t)\right]$, and the inspection technique being used [9].

$$
P_{\text {det }}=\Phi\left(\frac{\eta_{\text {str }}(t)-\eta_{0.5}}{\sigma_{\text {insp }}}\right)
$$

where $\Phi$ is the distribution function of the standard normal variable.

If a defect has been detected, the probability of making the repair $\left(P_{\text {rep }}\right)$ is calculated as indicated in [7], assuming that a repair will return the structure to its initial strength level. This assumption could be easily modified to return a structure to some specified percentage of its initial strength level after a repair. The specified percentage could be decreased over time, indicating the increasing difficulty of returning an aging structure to its initial strength level [9].

After inspection, a decision must be made regarding whether or not to repair the structure based on the degree of damage that was detected in the inspection. The repair decision made after the first inspection affects the later decisions. As the number of inspections $(n)$ increases, the number of decision paths increases by $2^{n}$. Fig. 2 illustrates these paths for four inspections during ten years using an event tree.

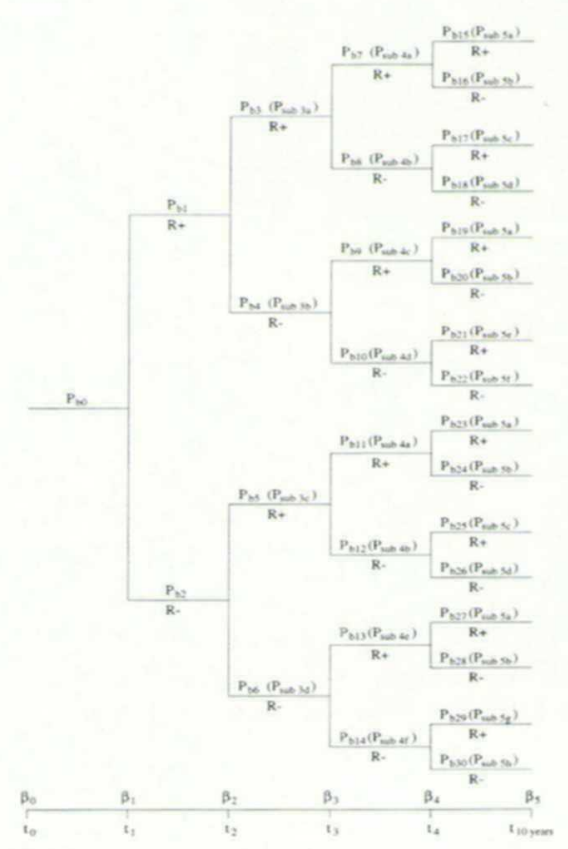

Fig. 2: Event tree for four inspections in ten years

Table 1: Main parameters of four inspection techniques 
The probability of taking path $i\left(P_{b_{i}}\right)$ is equal to

$$
P_{b_{i}}=\prod_{j=1}^{m} P_{\text {sub }}
$$

where $P_{s u b}$ is the probability of taking sub-branch $j$ along path $i$, and $m$ is the number of sub-branches. The probability of taking a sub-branch that involves making a repair $\left(R^{+}\right)$is equal to

$$
P_{\mathrm{sub}_{\mathrm{K}^{*}}}=P_{\mathrm{det}} P_{\mathrm{rep}}
$$

This probability accounts for the damage intensity and the ability of the chosen inspection technique to detect the damage. Similarly, the probability of taking any sub-branch where a repair is not made $\left(R^{-}\right)$is

$$
P_{\mathrm{sub}_{k^{-}}}=1-P_{\mathrm{sub}_{k^{*}}}
$$

For each Branch $_{i}$ on the event tree, the probability of failure of the structure given that Branch $_{i}$ was taken $\left[P_{f}(\right.$ StructurelBranch $\left.)\right]$ is multiplied by the probability of that branch being taken $\left(P_{b_{i}}\right)$. The lifetime probability of failure is equal to the sum of the product $\left[P_{f}\left(\right.\right.$ StructurelBranch $\left.\left.h_{i}\right) P_{b_{i}}\right]$ over all branches. Consequently, the lifetime reliability index $(\beta)$ can be found.

The cost of repair $\left(C_{\text {rep }}\right)$ is the sum of a fixed cost $\left(C_{\text {fix }}\right)$, which occurs every time a repair is made (i.e. planning, getting to the site, exposing the element), and a variable cost $\left(C_{\mathrm{var}}\right)$, which depends on the degree of damage (e.g. the amount of material that needs to be replaced). The expected

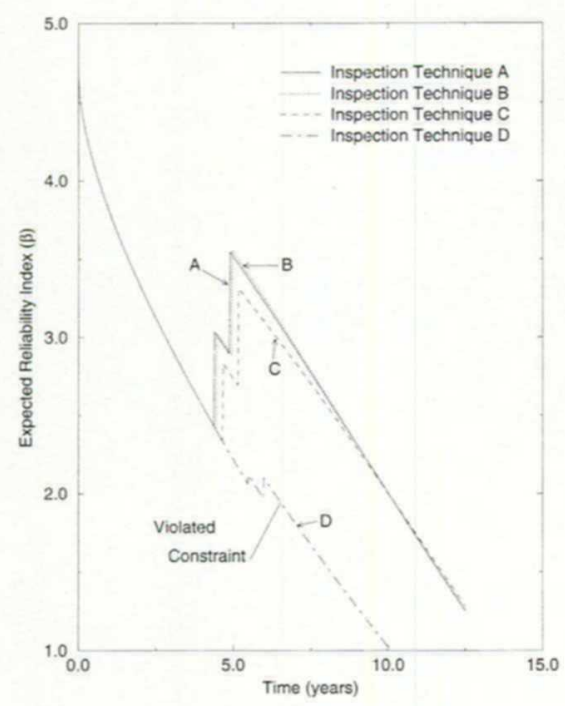

Fig. 3: Reliability Index over time for four inspection techniques considering two inspections in ten years total cost $\left[E\left(C_{\text {tot }}\right)\right]$ is the sum of the expected lifetime inspection cost $\left[E\left(C_{\text {insplife }}\right)\right]$ and the expected lifetime cost of repair $\left[E\left(C_{\text {replife }}\right)\right]$.

It is the expected total cost that will be minimized to find the optimal method of inspection and the optimal inspection times. In this example, the time value of money is not considered. A detailed description of every calculation step is shown in [7].

Using four lifetime inspections for the simple deteriorating structure assumed, the optimization problem can be formulated as:

Minimize $E\left(C_{\text {tot }}\right)$ such that

$\beta_{t 1} \geq 2.0 ; \beta_{t 2} \geq 2.0 ; \beta_{t 3} \geq 2.0 ;$

$\beta_{t 4} \geq 2.0 ; \beta_{10}$ years $\geq 2.0$

$0.5 \leq t_{1} \leq 7.0 ; 0.5 \leq t_{2}-t_{1} \leq 7.0 ;$

$0.5 \leq t_{3}-t_{2} \leq 7.0 ; 0.5 \leq t_{4}-t_{3} \leq 7.0$;

$t_{4} \leq 10.0$

where $t_{1}$ to $t_{4}$ are the times (in years) when the four inspections will be conducted. Eq. 7 ensures that the inspections are at least six months apart but not more than seven years apart. The optimizations for two and three lifetime inspections are formulated in a similar manner $[7,8]$.

Fig. 3 shows the expected reliability index over time for all four inspection techniques considering two lifetime inspections, $C_{\mathrm{fix}}=5.0$ and $C_{\mathrm{var}}=5.0 \eta_{\mathrm{rep}}$ [7]. As the quality of the inspection technique improved (A and D being the best and worst techniques, respectively), the optimum timing of the inspection was earlier in the life of the structure, and the improvement in the expected reliability index after an inspection was greater. The improvement in the expected reliability index indicates a higher probability of taking a path on the event tree that would lead to a repair. The expected cost was higher for the higher quality inspection techniques. The optimization problem could not be solved using inspection technique $\mathrm{D}$ without violating at least one constraint (Fig. 3).

The global optimum solution is determined by solving the problem for all inspection techniques for two, three, and four lifetime inspections. The expected total costs associated with all options are shown in Fig. 4, where it can be seen that the optimum solution is associated with two lifetime inspections using inspection technique $\mathrm{C}$. The optimal inspection times are 4.67

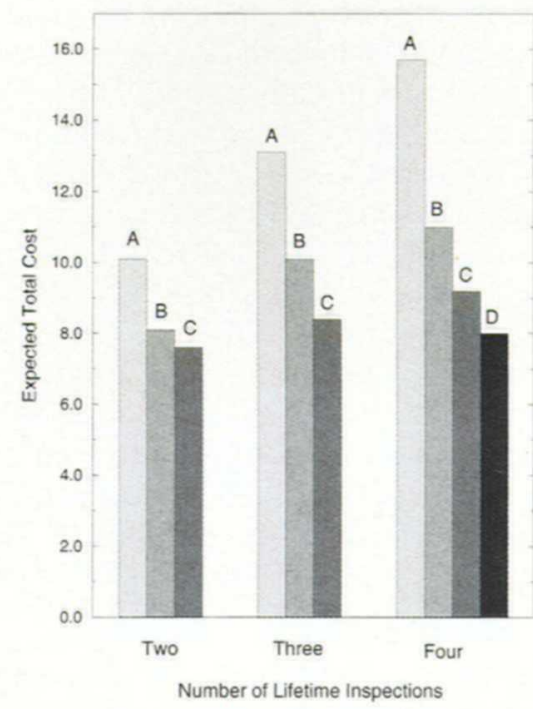

Fig. 4: Expected inspection/repair cost for four inspection techniques considering two, three and four inspections in ten years

years and 5.17 years, and the expected total cost was 7.6.

\section{Existing Bridges}

The proposed methodology was applied to an existing concrete bridge deck using the half-cell potential inspection method (Fig. 5) and realistic cost and inspection/repair data. The structure is a $42.1 \mathrm{~m} \times 12.2 \mathrm{~m}$ concrete bridge deck.

As salts are applied to the deck, chlorides penetrate the concrete. When the chloride concentration reaches a critical threshold concentration at the reinforcing steel, corrosion begins. This eventually causes spalls and delaminations in the concrete. The deck will be replaced when active corrosion is underway in at least $50 \%$ of the deck, consistent with Colorado Department of Transportation policy [10]. The mean chloride initiation time for the concrete deck was calculated as 19.60 years and the standard deviation as 7.51 years [7].

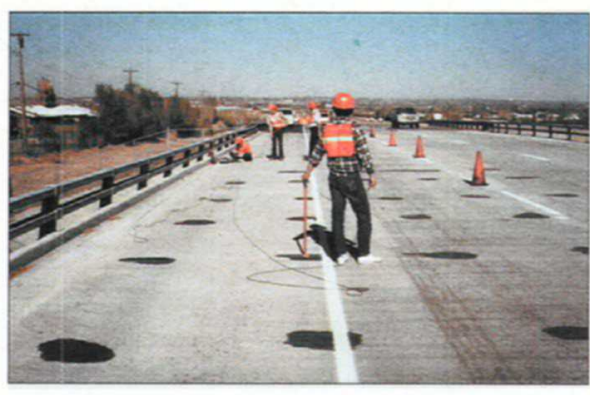

Fig. 5: Half-cell potential test (Photo courtesy of the Colorado Department of Transportation) 
The uncertainty of assessing the condition of the entire deck from a finite number of half-cell readings is considered using three inspection techniques ( $\mathrm{A}, \mathrm{B}$ and $\mathrm{C}$ ), where the differences are the spacings between half-cell readings $(1.52,3.05$ and $6.10 \mathrm{~m}$, for methods $\mathrm{A}, \mathrm{B}$ and $\mathrm{C}$, respectively) and inspection costs (1996 USD 1027, 604 and 408 , respectively). The inspection cost was estimated in consultation with specialists from the Colorado Department of Transportation. These costs include travel time to the site, traffic control, test set-up, recording readings, and preparing a final report [11].

The only repair option considered is replacement of the deck at a repair cost of USD 225600 [12]. The probability of making a repair is a function of the number of half-cell readings, the interpreted results of the inspection, and the bridge manager's approach to repair. Four repair approaches (delayed, linear, proactive and idealized) are used [7]. The repair approach relates the interpreted damage of the deck to the bridge manager's willingness to make the repair based on past performance.

A discrete optimization of the bridge deck was conducted for one, two, three and four lifetime inspections. For the case of four lifetime inspections, the optimization problem that minimizes the expected value of the total cost is formulated as:

Minimize $E\left(C_{\text {tot }}\right)$ such that

$$
E(\text { Damage }) \leq 0.50
$$

$2.0 \leq t_{1} \leq 20.0 ; 2.0 \leq t_{2}-t_{1} \leq 20.0 ;$ $2.0 \leq t_{3}-t_{2} \leq 20.0 ; 2.0 \leq t_{4}-t_{3} \leq 20.0$; $t_{4} \leq$ Life $_{\text {service }}$

Eq. 8 ensures that the expected damage of the deck $[E($ Damage $)]$ at any time never exceeds the $50 \%$ damage limit established by the replacement policy. Eq. 9 ensures that the inspections are at least 2 years apart but not more than 20 years apart.

After an inspection, a decision regarding whether or not to repair the structure based on the degree of damage detected is made using an event tree. The probability of taking any branch or sub-branch on the event tree is calculated using Eqs. 4 and 5.

The repair criterion in this example is based on the expected value of damage rather than on an expected reliability index. The expected damage computation is similar to that used for the lifetime reliability index computation. For each branch on the event tree, the expected damage to the structure given that Branch $_{i}$ was taken, $\left[E\left(\right.\right.$ Damage|Branch $\left.\left._{i}\right)\right]$ is multiplied by the probability of that branch being taken $\left(P_{b_{i}}\right)$. The total expected damage to the structure is equal to the sum over all branches.

The expected value of the total cost to be minimized is similar to that for simple structures. The difference is that the lifetime costs of inspection and repair are discounted back to the time at which the investment decisions were made using the discount rate, which accounts for monetary inflation.

Assuming a discount rate of $2 \%$, an expected service life of the bridge of 45 years, a proactive approach to repair, and inspection technique $\mathrm{A}$ (i.e. $C_{\text {insp }}=$ USD 1027), the optimum inspection strategy requires three inspections at $10.05,19.76$ and 35.45 years. The expected optimal total cost is USD 174 175. Fig. 6 shows the expected value of damage at each inspection and the expected effect of deck replacement. There appears to be little probability of replacing the deck after the first inspection, but a fairly high likelihood of replacement after the second and/or third inspection.

A higher discount rate would make the money to be earned or spent in the future worth less and will increase the benefit of making repairs later in the life of the structure. Table 2 shows the optimum inspection times and expected inspection and repair costs of a reinforced concrete bridge deck with an expected service life of 40 years with four lifetime inspections, a proactive

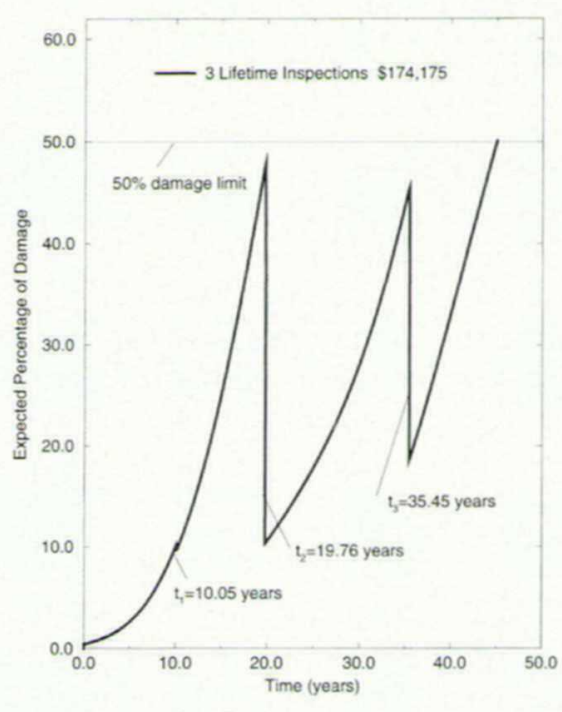

Fig. 6: Optimum inspection/repair program for an existing concrete bridge deck

approach to repair, and a $50 \%$ damage limit replacement policy for different discount rates.

The optimal strategy at $0 \%$ discount rate is expected to cost about USD 246000 (Table 2). With a discount rate of $10 \%$, the cost is about USD 35000 . Therefore, the discount rate has a significant effect on the optimum solution.

While the initial optimum strategy features three lifetime inspections at $10.05,19.76$ and 35.45 years, the inspection and repair program will be updated after each inspection to incorporate the new information that the inspection provides. After the first inspection, a replacement decision will be made, and four of the eight paths can be eliminated. With this additional information, an updated optimum inspection plan can be developed [7].

\begin{tabular}{|c|c|c|c|c|c|}
\hline \multirow{2}{*}{$\begin{array}{l}\text { Discount } \\
\text { rate ( } \%)\end{array}$} & \multicolumn{4}{|c|}{ Optimum inspection time (years) } & \multirow{2}{*}{$\begin{array}{l}\text { Minimum expected } \\
\text { cost (1996 USD) }\end{array}$} \\
\hline & $t_{1}$ & $t_{2}$ & $t_{3}$ & $t_{4}$ & \\
\hline 0 & 8.03 & 11.78 & 20.02 & 25.88 & 246050 \\
\hline 1 & 9.04 & 13.83 & 20.35 & 26.15 & 202110 \\
\hline 2 & 8.66 & 13.83 & 20.95 & 26.70 & 166250 \\
\hline 3 & 7.88 & 15.55 & 22.08 & 29.18 & 135170 \\
\hline 4 & 8.02 & 15.82 & 22.43 & 29.87 & 110660 \\
\hline 5 & 8.14 & 16.47 & 23.93 & 30.60 & 90579 \\
\hline 6 & 8.19 & 17.11 & 26.03 & 32.30 & 74117 \\
\hline 7 & 8.37 & 17.23 & 26.45 & 32.51 & 61082 \\
\hline 8 & 8.34 & 17.30 & 26.72 & 32.66 & 50572 \\
\hline 9 & 8.62 & 17.37 & 27.04 & 32.70 & 42077 \\
\hline 10 & 8.36 & 17.46 & 27.35 & 32.89 & 35206 \\
\hline
\end{tabular}

Table 2: Effect of discount rate on optimum inspection times and minimum expected lifetime inspection and repair costs of a 40-year-old bridge deck 


\section{Conclusions}

A general methodology was introduced for optimum lifetime planning of bridge inspection and repair programs based on reliability and cost. This methodology minimizes expected inspection and repair costs and maintains an expected level of lifetime performance for a deteriorating bridge. The result identifies the optimum inspection technique, and the optimum numbers and timing of lifetime inspections and repairs. An event tree accounts for all possible repair outcomes after the inspections, and the optimum strategy is based on the likelihood of following various paths on the event tree. The optimum strategy must be updated after every inspection as more information becomes available.

The methodology described here and results presented elsewhere $[7,8]$ offer a rational basis for optimum planning. Uncertainties associated with randomness and imperfect modeling and estimation must be considered and combined to obtain a robust optimum strategy. It is hoped that information obtained in the future will reduce uncertainties and, the cost of the strategy.

\section{References}

[1] THOFT-CHRISTENSEN, P.; SøRENSEN, J. D. Optimal strategy for inspection and repair of structural systems. Civil Engineering Systems, Vol. 4, 1987, pp. 94-100.

[2] MORI, Y.; ELLINGWOOD, B.R Maintaining reliability of concrete structures II: Optimum inspection/repair. Journal of Structural Engineering, Vol. 120, 1994, pp. 846-862.

[3] WEN, Y. K.; KANG, Y. J. Design based on minimum expected life-cycle cost. In: Advances in Structural Optimization (Frangopol, D. M.; Cheng, F. Y., Eds), ASCE, New York, 1997, pp. 192-203.

[4] FRANGOPOL, D. M. Probabilistic structural optimization. Progress in Structural Engineering and Materials, Vol. 1, 1998, pp. 223-230.

[5] DAS, P.C. Whole life performance based assessment of highway structures: Proposed procedure. In: Safety of Bridges (Das, P. C., Ed.), Thomas Telford, London, 1997, pp. 161-165.

[6] ZIMMERMAN, R.; SPARROW, R. Workshop on Integrated Research for Civil Infrastructure, Final Report to the National Science Foundation, New York University, NY, 1997.
[7] ESTES, A.C. A system reliability approach to the lifetime optimization of inspection and repair of highway bridges. $\mathrm{Ph}$.D. Thesis, University of Colorado, Boulder, CO, 1997.

[8] FRANGOPOL, D. M.; ESTES, A.C. Optimum design of bridge inspection/repair programs based on lifetime reliability and life-cycle cost. In: The Management of Highway Structures (Das, P.C., Ed.). Thomas Telford, London, 1999.

[9] FRANGOPOL, D.M.; LIN, K.-Y.; ESTES, A.C. Life-cycle cost design of deteriorating structures. Journal of Structural Engineering, Vol. 123, 1997, pp. 1390-1401.

[10] Colorado Department of Transportation. 1996 Bridge Deck Information and Testing, Denver, CO, 1996.

[11] Colorado Department of Transportation. 1995 Cost Data, Denver, CO, 1995.

[12] FRANGOPOL, D. M.; ESTES, A.C. Lifetime bridge maintenance strategies based on system reliability. Structural Engineering International, Vol. 7, 1997, pp. 193-198.

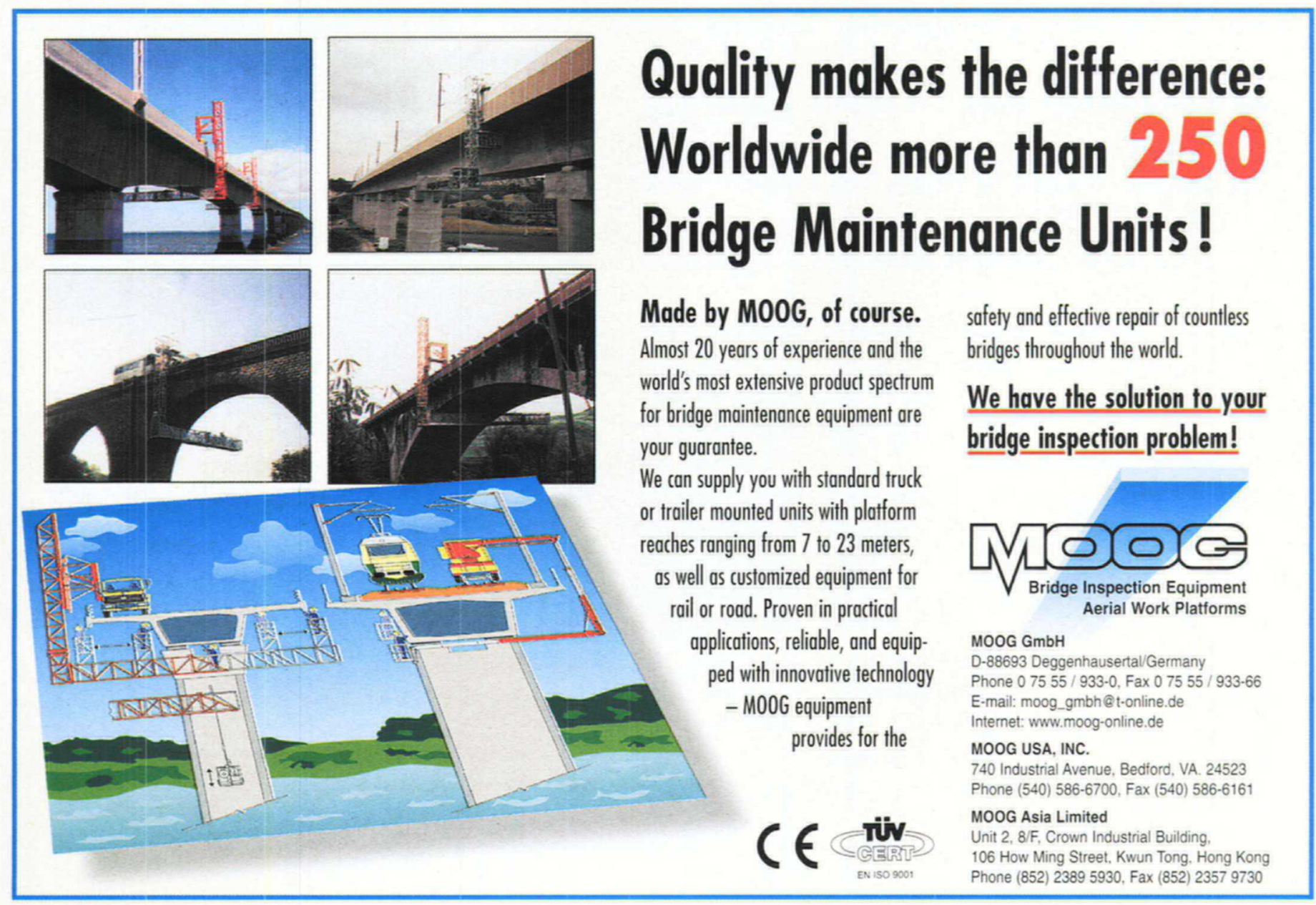

